\title{
Gravitational Radiation, Vorticity And Super-Energy: A Conspicuous Threesome
}

\author{
Luis Herrera \\ Instituto Universitario de Física Fundamental y Matemáticas, Universidad de Salamanca, \\ 37007 Salamanca, Spain; lherrera@usal.es
}

Received: 23 May 2019; Accepted: 1 July 2019; Published: 4 July 2019

\begin{abstract}
We elaborate on the link relating gravitational radiation, vorticity and a flux of super-energy on the plane orthogonal to the vorticity vector. We examine the vorticity appearing in the congruence of observers at the outside of the source, as well as the vorticity of the fluid distribution, the source of the gravitational radiation is made of. The information provided by the study of the physical aspects of the source poses new questions which could, in principle, be solved by the observational evidence. Besides the study of the theoretical issues associated to such relationship, we also stress the new observational possibilities to detect gravitational radiation, appearing as consequence of the above mentioned link. The high degree of development achieved in the gyroscope technology, as well as recent proposals to detect rotations by means of ring lasers, atom interferometers, atom lasers and anomalous spin-precession experiments, lead us to believe that an alternative to the laser interferometers used so far to detect gravitational waves, may be implemented based on the detection of the vorticity associated with gravitational radiation. Additionally, this kind of detectors might be able to elucidate the open question about the physical properties of the tail of the waves appearing as the consequence of the violation of the Huygens's principle in general relativity.
\end{abstract}

Keywords: vorticity; gravitational radiation; super-energy

PACS: 04.20.-q; 04.20.Cv; 04.30.NK

\section{Introduction}

The detection of gravitational radiation by means of laser interferometers represents a major breakthrough in the development of the theory of gravitation [1]. However, the apparatus involved in such experiments is extremely expensive and requires the participation of large number of researchers, as a consequence of which this observational approach is accessible only to huge collaboration groups endowed with very large budgets. Therefore it is clearly alluring the idea to detect gravitational radiation by means of experiments based on effects, different from those involved in laser interferometers, that could be carried out by means of a cheaper technology and thereby affordable to a larger number of groups of research all around the world.

Our purpose in this paper is twofold. On the one hand we shall present a comprehensive discussion about the link between gravitational radiation, vorticity and a flux of super-energy on the plane orthogonal to the vorticity. On the other hand we would like to call the attention to the potential observational application of this effect on the detection of gravitational radiation.

The relationship between gravitational radiation and vorticity was put forward for the first time in Reference [2], using the Bondi approach [3,4]. It was subsequently discussed by many researchers (see for example References [5-11] and references therein). Short afterwards, it was established that the link between gravitational radiation and vorticity becomes intelligible if we introduce another 
element into the discussion, namely a flux of super-energy on the plane orthogonal to the vorticity vector $[8,10]$.

The idea to relate the vorticity to a flow of super-energy, put forward in Reference [8], stems from an intriguing fact related to the space-time generated by a magnetic dipole plus a central charge. The point is that such space-time appears to be stationary (non-static), that is, there is a vorticity in the congruence of observers at rest with respect to the source. In order to explain this strange situation, Bonnor [12] invokes a curious result of classical electrodynamics concerning the Poynting vector of a charged magnetic dipole (see Reference [13]). Indeed, it appears that for a charged magnetic dipole there is a non-vanishing flow of electromagnetic energy (as described by the Poynting vector), in spite of the fact that the system is time-independent. Thus, Bonnor explains the appearance of vorticity in such a system, as produced by the circular flow of electromagnetic energy (later on it was established that this is the explanation for the appearance of vorticity in any stationary electrovacuum solution to the Einstein equations [14]). In Reference [8] we have just extended the Bonnor's idea by replacing the electromagnetic energy by super-energy, in order to explain the appearance of vorticity associated to the emission of gravitational radiation.

All the discussion above concerns the vorticity of the congruence of observers in the vacuum (outside the source). However a similar link exists between gravitational radiation and the vorticity of the congruence of the fluid elements forming the source. The combined discussion from the point of view of observers, both, outside and inside the source, provides a deeper insight into the problem and poses new questions which eventually could be answered by experimental observation.

Before entering into the core of the discussion it would be convenient to specify in some detail the three essential ingredients of our proposal.

\section{Vorticity, Gravitational Radiation and Super-Poynting Vector}

Let us start with the concept of vorticity of a congruence. As is well known the vorticity vector $\Omega$ defining the vorticity of a congruence, describes the rate of rotation (the proper angular velocity) with respect to proper time at any reference particle at rest in the rotating frame, relative to the local compass of inertia. This compass of inertia is physically realized by three gyroscopes spinning about three orthogonal axes. It is then obvious that $-\boldsymbol{\Omega}$ describes the rotation of the compass of inertia (gyroscope) with respect to reference particles. From these comments the suitability of gyroscopes to detect rotations becomes evident.

Two very important relativistic effects can be identified and measured by means of gyroscopes. One is the well known Fokker-de Sitter effect, which refers to the precession of a gyroscope following a closed orbit around a spherically symmetric mass distribution (a version of Thomas precession in the Schwarzschild spacetime). This effect can be verified with a great degree of accuracy by observing the rotation of the earth-moon system around the sun [15]. The other effect is the Lense-Thirring-Shiff precession, which is much more difficult to observe and refers to the generation of vorticity in the congruence of observers due to the rotation of the source (the so called frame dragging effect, see Reference [16] for a detailed discussion on this issue). The Gravity Probe B experiment not only confirmed the reality of the Lense-Thirring-Shiff precession $[17,18]$ but also put in evidence the high degree of development achieved so far in the gyroscope technology.

Next, let us say few words about super-energy and super-Poynting vector.

In any classical field theory, energy is a quantity defined in terms of potentials and their first derivatives. Accordingly, in general relativity a definition of energy through a tensor variable is impossible since, as is well known, we cannot form a tensor with the metric and its first derivatives alone. In other words, a local description of gravitational energy in terms of true invariants (tensors of any rank) is not possible within the context of the theory. This situation has lead researchers to look for non-local definitions of energy or to describe it by means of pseudo-tensors or to resort to a succedaneous definition of energy. 
Super-energy is an example of this last alternative. It may be defined from the Bel or the Bel-Robinson tensor [19-21] (they both coincide in vacuum) and has been shown to be very useful when it comes to explaining a number of phenomena in the context of general relativity.

The Bel and the Bel-Robinson tensors are obtained from the Riemann and the Weyl tensor (as well as their dual) respectively, by analogy with the form in which the energy-momentum tensor of the electromagnetic field depends on the Maxwell tensor (and its dual). Obviously they coincide in vacuum but differ within the source. Also, by analogy with electromagnetism, a super-Poynting vector describing the flux of super-energy can be defined. This quantity is particularly relevant for our discussion since in the theory of the super-Poynting vector, a state of gravitational radiation is associated to a non-vanishing component of the latter (see References [19-21]). This is in agreement with the established link between the super-Poynting vector and the news functions [8], in the context of the Bondi-Sachs approach [3,4] (see below).

Finally, we shall provide a summarized description of gravitational radiation. This will be done for, both, the space-time outside the source, which we consider bounded in order for the spacetime to be asymptotically flat, as well as for the space-time within the source.The following two subsections are devoted to these issues.

\subsection{Gravitational Radiation in An Asymptotically Flat Vacuum Space-Time: The Bondi Approach}

The Bondi formalism [3] is centered around the general form of an axially and reflection symmetric asymptotically flat metric given by (for the general case see Reference [4])

$$
\begin{aligned}
d s^{2} & =\left(\frac{V}{r} e^{2 \beta}-U^{2} r^{2} e^{2 \gamma}\right) d u^{2}+2 e^{2 \beta} d u d r \\
& +2 U r^{2} e^{2 \gamma} d u d \theta-r^{2}\left(e^{2 \gamma} d \theta^{2}+e^{-2 \gamma} \sin ^{2} \theta d \phi^{2}\right),
\end{aligned}
$$

where $V, \beta, U$ and $\gamma$ are functions of $u, r$ and $\theta$.

The coordinates are numbered $x^{0,1,2,3}=u, r, \theta, \phi$, respectively. $u$ is a timelike coordinate $\left(g_{u u}>0\right)$ and the hypersurfaces $u=$ constant define null surfaces (their normal vectors are null vectors), which in the null infinity $(r \rightarrow \infty)$ coincide with the Minkowski null light cone open to the future. $r$ is a null coordinate $\left(g_{r r}=0\right)$ and $\theta$ and $\phi$ are two angle coordinates (see Reference [3] for details).

Regularity conditions in the neighborhood of the polar axis $(\sin \theta=0)$, imply that as $\sin \theta \rightarrow 0$

$$
V, \beta, U / \sin \theta, \gamma / \sin ^{2} \theta,
$$

each equals a function of $\cos \theta$ regular on the polar axis.

Expanding the metric functions in series of $1 / r$, and using the field equations one gets

$$
\begin{gathered}
\gamma=c r^{-1}+\left(C-\frac{1}{6} c^{3}\right) r^{-3}+\mathcal{O}\left(r^{-n}\right) \quad n \geq 4, \\
U=-\left(c_{\theta}+2 c \cot \theta\right) r^{-2}+\left[2 N+3 c c_{\theta}+4 c^{2} \cot \theta\right] r^{-3}+\mathcal{O}\left(r^{-n}\right) \quad n \geq 4, \\
V=r-2 M-\left(N_{\theta}+N \cot \theta-c_{\theta}^{2}-4 c c_{\theta} \cot \theta-\frac{1}{2} c^{2}\left(1+8 \cot ^{2} \theta\right)\right) r^{-1}+\mathcal{O}\left(r^{-n}\right) \quad n \geq 2, \\
\beta=-\frac{1}{4} c^{2} r^{-2}+\mathcal{O}\left(r^{-n}\right) \quad n \geq 3,
\end{gathered}
$$

where letters as subscripts denote derivatives and $c, C, N$ and $M$ are functions of $u$ and $\theta$ satisfying the constraint

$$
4 C_{u}=2 c^{2} c_{u}+2 c M+N \cot \theta-N_{\theta} .
$$


The three functions $c, M$ and $N$ are further constrained by the supplementary conditions

$$
\begin{gathered}
M_{u}=-c_{u}^{2}+\frac{1}{2}\left(c_{\theta \theta}+3 c_{\theta} \cot \theta-2 c\right)_{u}, \\
-3 N_{u}=M_{\theta}+3 c c_{u \theta}+4 c c_{u} \cot \theta+c_{u} c_{\theta} .
\end{gathered}
$$

In the static case $M$ is related to the mass of the system and was called by Bondi the "mass aspect", whereas $N$ and $C$ are closely related to the dipole and quadrupole moment respectively.

Using the mass aspect, Bondi defines the mass $m(u)$ of the system as

$$
m(u)=\frac{1}{2} \int_{0}^{\pi} M \sin \theta d \theta,
$$

which, using (5) and (2) produces

$$
m_{u}=-\frac{1}{2} \int_{0}^{\pi} c_{u}^{2} \sin \theta d \theta .
$$

We may now summarize the more relevant results emerging from the Bondi approach:

- If $\gamma, M$ and $N$ are known on some null hypersurface $u=a$ (constant) and $c_{u}$ (the news function) is known for all $u$ in the interval $a \leq u \leq b$, then the system is fully determined in that interval. This implies that whatever happens at the source, leading to changes in the field, it can only do so by affecting $c_{u}$ and viceversa. This result establishes in an unmistakable way the relationship between news function and the occurrence of radiation.

- As it follows from (8), the mass of a system is constant if and only if there are no news.

Now, for an observer at rest in the frame of (1), the four-velocity vector has components

$$
V^{\alpha}=\left(\frac{1}{A}, 0,0,0\right),
$$

with

$$
A \equiv\left(\frac{V}{r} e^{2 \beta}-U^{2} r^{2} e^{2 \gamma}\right)^{1 / 2} .
$$

For the observer defined by (9) the vorticity vector may be written as (see Reference [2] for details)

$$
\omega^{\alpha}=\left(0,0,0, \omega^{\phi}\right),
$$

while for its absolute value we obtain

$$
\begin{aligned}
\Omega & \equiv\left(-\omega_{\alpha} \omega^{\alpha}\right)^{1 / 2}=-\frac{1}{2 r}\left(c_{u \theta}+2 c_{u} \cot \theta\right) \\
& +\frac{1}{r^{2}}\left[M_{\theta}-M\left(c_{u \theta}+2 c_{u} \cot \theta\right)-c c_{u \theta}+6 c c_{u} \cot \theta+2 c_{u} c_{\theta}\right]+\mathcal{O}\left(r^{-n}\right) \quad n \geq 3 .
\end{aligned}
$$

The first term on the right hand side of (12) describes the contribution of gravitational radiation to vorticity, it vanishes if and only if there are no news, that is, if there is no gravitational radiation. Instead, the second term survives even if $c_{u}=0$. In this latter case the vorticity has to be related to the tail of the wave associated to the $M_{\theta}$ term. We shall discuss further on this issue in the last section.

Finally, we shall need an expression for the super-Poynting vector. The super-Poynting vector based on the Bel-Robinson tensor is defined as (see Reference [8] for details)

$$
P_{\alpha}=\eta_{\alpha \beta \gamma \delta} E_{\rho}^{\beta} H^{\gamma \rho} u^{\delta},
$$

where $\eta_{\alpha \beta \gamma \delta}$ is the permutation symbol and $E_{\mu v}$ and $H_{\mu v}$, are the electric and magnetic parts of Weyl tensor, respectively. They are defined from the Weyl tensor $C_{\alpha \beta \gamma \delta}$ and its dual $\tilde{C}_{\alpha \beta \gamma \delta}$ by contraction with the four velocity vector, as

$$
E_{\alpha \beta}=C_{\alpha \gamma \beta \delta} V^{\gamma} V^{\delta}
$$




$$
H_{\alpha \beta}=\tilde{C}_{\alpha \gamma \beta \delta} u^{\gamma} u^{\delta}=\frac{1}{2} \eta_{\alpha \gamma \epsilon \delta} C_{\beta \rho}^{\epsilon \delta} V^{\gamma} V^{\rho}
$$

For the Bondi metric we obtain

$$
P_{\mu}=\left(0, P_{r}, P_{\theta}, 0\right)
$$

where the leading terms of each component are

$$
\begin{gathered}
P_{r}=-\frac{2 c_{u u}^{2}}{r^{2}}+\mathcal{O}\left(r^{-n}\right) \quad n \geq 3, \\
P_{\theta}=-\frac{2}{r^{2} \sin \theta}\left[\left(2 c_{u u}^{2} c+c_{u u} c_{u}\right) \cos \theta+\left(c_{u u} c_{\theta u}+c_{u u}^{2} c_{\theta}\right) \sin \theta\right]+\mathcal{O}\left(r^{-n}\right) \quad n \geq 3 .
\end{gathered}
$$

Thus, we have a radial component describing the propagation of super-energy along the generators of the null hypersurface $u=$ constant and a "meridional" component which is the one related to the vorticity (see References $[8,10]$ for details). A striking confirmation of the link between vorticity and and a flow of super-energy on the the plane orthogonal to the vorticity vector is provided by analyzing the Einstein-Rosen metric. In this case there is a radial component of the Super-Poynting vector related to the propagation of the gravitational wave, whereas the absence of vorticity is explained by the fact that there is no flow of super-energy in any plane of the 3-space (see References [10] for details). The vanishing of the $\phi$-component is due to the reflection symmetry of the Bondi metric.

We shall next provide a brief description of the the source of the gravitational radiation.

\subsection{Gravitational Radiation within the Source}

The Bondi approach sketched in the previous subsection is very powerful to analyze the radiative space-time outside the source but fails as we approach the source, due to the appearance of caustics, and of course is not suitable for describing the situation within the source. Accordingly in order to describe the situation within the fluid distribution we shall resort to a different framework, developed in Reference [22] and based on the $1+3$ approach [23-26], whose main characteristics are described below (for details see Reference [22]).

Thus, let us consider, axially (and reflection) symmetric sources, for which the most general line element may be written as:

$$
d s^{2}=-A^{2} d t^{2}+B^{2}\left(d r^{2}+r^{2} d \theta^{2}\right)+C^{2} d \phi^{2}+2 G d \theta d t
$$

where $A, B, C, G$ are positive functions of $t, r$ and $\theta$. We number the coordinates $x^{0}=t, x^{1}=r, x^{2}=\theta$, $x^{3}=\phi$.

The source is filled with an anisotropic and dissipative fluid, therefore the energy momentum tensor may be written in the "canonical" form, as

$$
T_{\alpha \beta}=(\mu+P) V_{\alpha} V_{\beta}+P g_{\alpha \beta}+\Pi_{\alpha \beta}+q_{\alpha} V_{\beta}+q_{\beta} V_{\alpha}
$$

where $\mu$ is the energy density, $q_{\alpha}$ is the heat flux, whereas $P$ is the isotropic pressure and $\Pi_{\alpha \beta}$ is the anisotropic tensor. We are considering an Eckart frame where fluid elements are at rest.

For the line element (19), it can be shown that the heat flux vector is determined by two scalar functions, whereas three scalar functions describe the anisotropic tensor. Also, there is a vorticity vector, with a single component along the $\phi$ direction, defined in terms of a single scalar function $\Omega$ given by

$$
\Omega=\frac{G\left(\frac{G, r}{G}-\frac{2 A, r}{A}\right)}{2 B \sqrt{A^{2} B^{2} r^{2}+G^{2}}} .
$$

Regularity conditions at the centre imply that: $G=0 \Leftrightarrow \Omega=0$. 
Finally we shall need to consider the super-Poynting vector $P^{\mu}$ within the source. As we know from Reference [8], there is always a non-vanishing component of $P^{\mu}$, on the plane orthogonal to a unit vector along which there is a non-vanishing component of vorticity (the $\theta-r$ plane). Instead, $P^{\mu}$ vanishes along the $\phi$-direction since there are no motions along this latter direction, because of the reflection symmetry.

Explicit expressions for the components of the super-Poynting vector may be found in Reference [22]. Suffice is to say at this point that we can identify three different contributions in the super-Poynting vector. On the one hand we have contributions from the heat transport process. These are in principle independent of the magnetic part of the Weyl tensor, which explains why (some of them) remain in the spherically symmetric limit. On the other hand we have contributions from the magnetic part of the Weyl tensor. These are of two kinds. On the one hand, contributions associated with the propagation of gravitational radiation within the fluid and on the other hand, contributions of the flow of super-energy associated with the vorticity on the plane orthogonal to the direction of propagation of the radiation, this is the effect relevant for our discussion here. Both contributions are intertwined and it appears to be impossible to disentangle them through two independent scalars.

Before concluding this section, the following remarks are in order:

- The gravitational radiation being a dissipative process, we should expect that an entropy generator factor be present in the source of radiation. This has been discussed with some detail in Reference [27].

- As a consequence of the previous point, the exterior of a gravitationally radiating source is necessarily filled with a null fluid, produced by the dissipative processes inherent to the emission of gravitational radiation. In other words the assumption of vacuum in the Bondi approach has to be regarded as an approximation, see Reference [28] for a discussion on this issue.

- It is important to keep in mind the difference between the steady vorticity of the stationary case (e.g., the one of the Kerr metric) and the vorticity considered here. In the former case there is no gravitational radiation, although the vorticity is also related to a flux of super-energy on the plane orthogonal to the vorticity vector (see Reference [29] for details).

- It is important to stress that we are dealing here, exclusively, with sources of gravitational radiation represented by a fluid distribution. In other words the emission of gravitational radiation is entirely due to changes in their relativistic multipole moments. Accordingly, we are excluding gravitational radiation of the "synchrotron" type produced by accelerated massive particles or the two body problem.

We have now available all elements we need for our discussion.

\section{Discussion}

The main point to retain from the preceding sections is that whenever gravitational radiation is emitted we should expect the appearance of vorticity in the congruence of the world lines of observers. Accordingly, any experimental device intended to measure rotations could be a potential detector of gravitational radiation as well. Of course, extremely high sensitivities have to be reached, for these detectors to be operational. However, even if the present technology might not be up to the required sensitivities, the intense activity deployed in recent years in this field, invoking ring lasers, atom interferometers, atom lasers, anomalous spin-precession, trapped atoms and quantum interference (see References [30-41] and references therein), besides the incredible sensitivities obtained so far in gyroscope technology and exhibited in the Gravity Probe B experiment $[17,18]$, make us confident in that this kind of detectors may be operating in the foreseeable future.

As already mentioned in the Introduction, the association of gravitational radiation and vorticity was first put in evidence from the study of the space-time outside the source. However, and this is perhaps one of our main points in this work, the information provided by the inclusion of the physical properties of the source into the whole picture, leads to new fundamental questions which could be answered by the observational evidence. 
Indeed, in Reference [42] an exhaustive analysis of axially symmetric fluid distributions just after its departure from equilibrium, has been carried out at the smallest time scale at which we can detect signs of dynamical evolution. It was then obtained that the departure from equilibrium and the ensuing evolution of all variables, is controlled by a single function-called the fluid news function-in analogy with the Bondi's news function. Such a function is related to the time derivative of the vorticity vector, putting in evidence the link between vorticity and radiation within the source.

However, the most relevant result obtained from this study, concerning the present discussion, is the fact that both the magnetic part of the Weyl tensor and $\Omega$ vanish at the time scale under consideration, whereas their first time derivatives are non-vanishing at that same time scale, thereby suggesting that both phenomena (radiation and vorticity), as well as the non-vanishing component of the super-Poynting vector on the plane orthogonal to the vorticity, occur essentially simultaneously. This is at variance with the point of view assumed in Reference [8] where it was assumed that radiation precedes the appearance of vorticity. Although this point deserves further discussion from the theoretical point of view, we hope that it could eventually be elucidated by experimental observation.

Another important issue appearing from the study of the physical properties of the source, concerns the tail of the gravitational wave. Indeed, as it is apparent from (12), the vorticity related to gravitational radiation combines two different type of contributions. On the one hand we have the term of order $\mathcal{O}\left(r^{-1}\right)$ which is directly related to the non-vanishing of the news function, that is, it is the vorticity associated to the emission of gravitational radiation. On the other hand, the term of $\mathcal{O}\left(r^{-2}\right)$ does not vanish after the emission of gravitational radiation stops $\left(c_{u}=0\right)$ and therefore has to be related to the tail of the wave, appearing as consequence of the violation of the Huygens's principle in curved space-times. Therefore the transition from a radiating regime to the static one seems to be forbidden and may happen only asymptotically in time. However, when we take into account the source of the gravitational radiation, it appears that the above conclusion is not evident. Let us analyze this issue more closely.

The appearance of tails after the emission of gravitational radiation has been established in studies considering exclusively the space-time outside the source and as a matter of fact far from the source (see References [43-49] and references therein). However, a recent study on the transition of a gravitationally radiating fluid source to equilibrium [50], shows that such a transition may take place at time scale of the order of thermal relaxation time, thermal adjustment time or hydrostatic time (whichever is larger). The explanation for such disagreement might be found in the fact that in the studies carried on in the vacuum, some physical phenomena describing the interaction of the field with the source might have been overlooked. This result strengthens further the importance to analyze the problem, from both the outside and the inside of the source. At any rate it is clear that observational detection of the $\mathcal{O}\left(r^{-2}\right)$ term in the vorticity, would help to clarify this point.

Finally, the following remark is in order: The presented discussion was carried out in the context of general relativity, however, as is known, due to several astronomical observations at different scales, which pose some problems of interpretation within the context of the "classical" Einstein theory, some researchers have found it useful to modified the general relativity in order to accommodate the above mentioned observational data. The interest on such alternative theories is therefore fully justified and the obvious question arises: how would the presented results change if, instead of using GR, we use any of the alternative theories? Although a thorough answer to the above question is out of the scope of this work, it seems very likely that the additional terms corresponding to these theories should appear in the expression for the vorticity and therefore the observational evidence could help to discriminate between different alternatives.

Funding: This research received no external funding.

Conflicts of Interest: The authors declare no conflict of interest. 


\section{References}

1. Abbott, B.P.; Abbott, R.; Abbott, T.D.; Abernathy, M.R.; Acernese, F.; Ackley, K.; Adams, C.; Adams, T.; Addesso, P.; Adhikari, R.X.; et al. Observation of Gravitational Waves from a Binary Black Hole Merger. Phys. Rev. Lett. 2016, 116, 061102. [CrossRef] [PubMed]

2. Herrera, L.; Hernández-Pastora, J.L. On the influence of gravitational radiation on a gyroscope. Class. Quantum Grav. 2000, 17, 3617-3625. [CrossRef]

3. Bondi, H.; van der Burg, M.G.J.; Metzner, A.W.K. Gravitational waves in general relativity VII. Waves from axi-symmetric isolated systems. Proc. Roy. Soc. A 1962, 269, 21-52.

4. Sachs, R. Gravitational waves in general relativity VIII. Waves in asymptotically flat space-time. Proc. Roy. Soc. A 1962, 270, 103-125.

5. Sorge, F.; Bini, D.; de Felice, F. Gravitational waves, gyroscopes and frame dragging. Class. Quantum Grav. 2001, 18, 2945-2958. [CrossRef]

6. Valiente, J. Can one detect a non-smooth null infinity? Class. Quantum Grav. 2001, 18, 4311-4316.

7. Herrera, L.; Santos, N.O.; Carot, J. Gravitational radiation, vorticity and the electric and magnetic part of Weyl tensor. J. Math. Phys 2006, 47, 052502. [CrossRef]

8. Herrera, L.; Barreto, W.; Carot, J.; di Prisco, A. Why does gravitational radiation produces vorticity? Class. Quantum Grav. 2007, 24, 2645-2651. [CrossRef]

9. Bicak, J.; Katz, J.; Lynden-Bell, D. Gravitational waves and dragging effects. Class. Quantum Grav. 2008, 25, 165017. [CrossRef]

10. Herrera, L. Radiation and vorticity: The missing link. Gen. Relativ. Gravit. 2014, 46, 1654-1657. [CrossRef]

11. Bini, A.D. Geralico and W. Plastino. Cylindrical gravitational waves: C-energy, super-energy and associated dynamical effects. Class. Quantum Grav. 2019, 36, 095012. [CrossRef]

12. Bonnor, W.B. Dragging of inertial frames by a charged magnetic dipole. Phys. Lett. A 1991, 158, $23-26$. [CrossRef]

13. Feynman, R.P.; Leighton, R.B.; Sand, M. Lectures on Physics II; Addison-Wesley: Reading, PA, USA, 1964; pp. 27-28.

14. Herrera, L.; González, G.A.; Pachón, L.A.; Rueda, J.A. Frame dragging, vorticity and electromagnetic fields in axially symmetric stationary spacetimes. Class. Quantum Grav. 2006, 23, 2395-2408. [CrossRef]

15. Shapiro, I.I.; Reasenberg, R.D.; Chandler, J.F.; Babcock, R.W. Measurement of the de Sitter precession of the Moon: A relativistic three-body effect. Phys. Rev. Lett. 1988, 61, 2643. [CrossRef]

16. Rindler, W.; Perlick, V. Rotating coordinates as tools for calculating circular geodesics and gyroscopic precession. Gen. Rel. Grav. 1990, 22, 1067-1081. [CrossRef]

17. Everitt, C.W.F.; DeBra, D.B.; Parkinson, B.W.; Turneaure, J.P.; Conklin, J.W.; Heifetz, M.I.; Keiser, G.M.; Silbergleit, A.S.; Holmes, T.; Kolodziejczak, J.; et al. Gravity Probe B: Final Results of a Space Experiment to Test General Relativity. Phys. Rev. Lett. 2011, 106, 221101. [CrossRef] [PubMed]

18. Ciufolini, I.; Pavlis, E. A confirmation of the general relativistic prediction of the Lense-Thirring effect. Nature 2004, 431, 958-960. [CrossRef] [PubMed]

19. Bel, L. Sur la radiation gravitationelle. C. R. Acad. Sci. 1958, 247, 1094-1096.

20. Bel, L. Radiation states and the problem of energy in general relativity. Gen. Rel. Grav. 2000, 32, $2047-2078$. [CrossRef]

21. Bel, L. Introduction d'un tenseur du quatrieme order. C. R. Acad. Sci. Paris 1959, 248, 1297-1300.

22. Herrera, L.; Di Prisco, A.; Ibáñez, J.; Ospino, J. Dissipative collapse of axially symmetric, general relativistic, sources: A general framework and some applications. Phys. Rev. D 2014, 89, 084034. [CrossRef]

23. Ellis, G.F.R. Relativistic Cosmology. In Proceedings of the International School of Physics "Enrico Fermi", Course 47: General Relativity and Cosmology; Sachs, R.K., Ed.; Academic Press: New York, NY, USA, 1971.

24. Ellis, G.F.R.; van Elst, H. Cosmological models (Cargese lectures 1998). arXiv 1998, arXiv:gr-qc/9812046.

25. Ellis, G.F.R. Republication of: Relativistic cosmology. Gen. Rel. Grav. 2009, 41, 581-660. [CrossRef]

26. Ellis, G.F.R.; Maartens, R.; MacCallum, M.A.H. Relativistic Cosmology; Cambridge University Press: Cambridge, UK, 2012.

27. Herrera, L.; di Prisco, A.; Ospino, J.; Carot, J. Shearing and geodesic axially symmetric perfect fluids that do not produce gravitational radiation. Phys. Rev. D 2015, 91, 024010. [CrossRef] 
28. Herrera, L.; di Prisco, A.; Ospino, J. The spacetime outside a source of gravitational radiation: The axially symmetric null fluid. Eur. Phys. J. C 2016, 94, 603-609. [CrossRef]

29. Herrera, L.; di Prisco, A.; Carot, J. Frame dragging and super-energy. Phys. Rev. D 2007, 76, 044012. [CrossRef]

30. Scully, M.O.; Zubairy, M.S.; Haugan, M.P. Proposed optical test of metric gravitation theories. Phys. Rev. A 1981, 24, 2009. [CrossRef]

31. Lenef, A.; Hammond, T.D.; Smith, E.T.; Chapman, M.S.; Rubenstein, R.A.; Pritchard, D.E. Rotation Sensing with an Atom Interferometer. Phys. Rev. Lett. 1997, 78, 760. [CrossRef]

32. Gustavson, T.L.; Bouyer, P.; Kasevich, M.A. Precision Rotation Measurements with an Atom Interferometer Gyroscope. Phys. Rev. Lett. 1997, 78, 2046. [CrossRef]

33. Stedman, G.E.; Schreiber, K.U.; Bilger, H.R. On the detectability of the Lense-Thirring field from rotating laboratory masses using ring laser gyroscope interferometers. Class. Quantum Grav. 2003, 20, 2527-2540. [CrossRef]

34. Stedman, G.E.; Hurst, R.B.; Schreiber, K.U. On the potential of large ring lasers. Opt. Commun. 2007, 279, 124-129. [CrossRef]

35. Di Virgilio, A.; Schreiber, K.U.; Gebauer, A.; Wells, J.-P.R.; Tartaglia, A.; Belfi, J.; Beverini, N.; Ortolan, A. A laser gyroscope system to detect the gravitomagnetic effect on earth. Int. J. Mod. Phys. D 2010, 19, 2331-2343. [CrossRef]

36. Bosi, F.; Cella, G.; di Virgilio, A.; Ortolan, A.; Porzic, A.; Solimenof, S.; Cerdonio, M.; Zendri, J.P.; Allegrini, M.; Bel, J.; et al. Measuring gravito-magnetic efects by multiring-laser gyroscope. Phys. Rev. D 2011, 84, 122002. [CrossRef]

37. Robins, N.P.; Altin, P.A.; Debs, J.E.; Close, J.D. Atom lasers: Production, properties and prospects for precision inertial measurement. arXiv 2012, arXiv:1209.2172v1.

38. Campbell, W.C.; Hamilton, P. Rotation sensing with trapped ions. arXiv 2016, arXiv:1609.00659v1.

39. Tartaglia, A.; di Virgilio, A.; Belf, J.; Beverini, N.; Ruggiero, M.L. Testing general relativity by means of ringlasers. Eur. Phys. J. Plus 2016, 132, 73. [CrossRef]

40. di Virgilio, A.; Beverini, N.; Carelli, G.; Ciampini, D.; Fuso, F.; Maccioni, E. Analysis of ring lasers gyroscopes including laser dynamics. arXiv 2019, arXiv:1904.02533v1.

41. Bonder, Y.; Herrera-Flores, J.E. Measuring Relativistic Dragging with Quantum Interference. arXiv 2019, arXiv:1905.02275v1.

42. Herrera, L.; di Prisco, A.; Ospino, J.; Carot, J. Earliest stages of the nonequilibrium in axially symmetric, self-gravitating, dissipative fluids. Phys. Rev. D 2016, 94, 064072. [CrossRef]

43. DeWitt, B.S.; Brehme, R. Radiation damping in a gravitational field. Ann. Phys. 1960, 9, 220-259. [CrossRef]

44. Kundt, W.; Newman, E.T. Hyperbolic Differential Equations in Two Dimensions. J. Math. Phys. 1968, 9 , 2193-2210. [CrossRef]

45. Bonnor, W.B. Approximate methods and gravitational radiation. In Proceedings of the Meeting on General Relativity; Barbera, G., Ed.; Comitato Nazionale per le Manifestazione Celebrative: Florence, Italy, 1965; pp. 119-142.

46. Couch, W.; Torrence, R.; Janis, A.; Newman, E.T. Tail of a Gravitational Wave. J. Math. Phys. 1968, 9, 484. [CrossRef]

47. Bonnor, W.B. Gravitational wave tails. In Gravitational Waves and Radiations, Proceedings of the International Conference, Paris, France, 18-22 June 1973; Transactions. (A75-26747 11-90); Centre National de la Recherche Scientifique: Paris, France, 1974; pp. 73-81.

48. Blanchet, L.; Shafer, G. Gravitational wave tails and binary star systems. Class. Quantum Grav. 1993, 10, 2699-2722. [CrossRef]

49. Marchand, T.; Blanchet, L.; Faye, G. Gravitational-wave tail effects to quartic non-linear order. Class. Quantum Grav. 2016, 33, 244003. [CrossRef]

50. Herrera, L.; di Prisco, A.; Ospino, J. The transition of a gravitationally radiating, dissipative fluid, to equilibrium. Can. J. Phys. 2018, 96, 1010-1015. [CrossRef]

(C) 2019 by the author. Licensee MDPI, Basel, Switzerland. This article is an open access article distributed under the terms and conditions of the Creative Commons Attribution (CC BY) license (http:/ / creativecommons.org/licenses/by/4.0/). 